\title{
Elle KOTTA
}

\section{ON THE INVERSE OF A SPECIAL CLASS OF MIMO BILINEAR SYSTEMS}

OLLE KOTTA. TEATUD TOOPI MITME SISENDI JA VALJUNDIGA BILINEAARSE SUSTEEMI POORDSOSTEEM

ЮЛЛЕ КОТТА. ОБ ОБРАТНОП СИСТЕМЕ СПЕЦИАЛЬНОГО КЛАССА БИЛИНЕННЫХ СИСТЕМ С МНӦГИМИ ВХОДАМИ И ВЫХОДАМИ

\section{(Presented by N. Alumäe)}

1. Introduction. The inverse system of a bilinear system (BLS) is, in general, a highly nonlinear system $\left[{ }^{1-3}\right]$. In $\left[{ }^{4}\right]$ the design of inverse system for a special class of single input-single output bilinear systems (BLS) has been examined. It has been shown that if the rank of the input matrix $B$ is unity, then the inverse system of a BLS is a linear time-invariant system and the complete controllability of the original system implies the complete controllability of the inverse system. The purpose of this paper is to generalize these results to multi input-multi output (MIMO) BLS. Unlike $\left[{ }^{4}\right]$, the discrete time systems are considered.

2. Problem statement. Consider the MIMO BLS described by the equations

$$
\begin{gathered}
x(t+1)=A x(t)+\sum_{i=1}^{m} B_{i} x(t) u_{i}(t), \\
y(t)=C x(t),
\end{gathered}
$$

where $x(t)$ is the $n$-dimensional state vector, $u_{i}(t), i=1, \ldots, m$ are the scalar controls, and $y(t)$ is the $m$-dimensional measurement vector. The matrices $A, B_{i}(i=1, \ldots, m), C$ are real constant matrices of appropriate dimensions and

$$
\operatorname{rank} B_{i}=1, \quad i=1, \ldots, m .
$$

Consider another system in the form

$$
\begin{aligned}
\tilde{x}(t+1) & =\tilde{A} \tilde{x}(t)+\widetilde{B} \tilde{u}(t) . \\
\tilde{y}(t) & =H(\tilde{x}(t), \tilde{u}(t)),
\end{aligned}
$$

where $\tilde{x}(t)$ is the $n$-dimensional state vector, $\tilde{u}(t)$ is the $m$-dimensional control vector and $\tilde{y}(t)$ is the $m$-dimensional output vector. The matrices $\tilde{A}, \widetilde{B}$ are constant matrices of appropriate dimensions and $H$ is a nonlinear map.

Def in ition. The system (4) is called inverse for the BLS (1)-(3) if, when driven by the output of the original system $y(t+1)$, it produces the input $u(t)$ of the original system as an output.

The purpose of this paper is to construct an inverse system for the BLS (1) - (3), i. e. to find the matrices $\widetilde{A}, \widetilde{B}$ and the form of the map $H$ :

3. Construction of inverse system. We shall now derive the equations for the inverse system. If to equations (2) unit shift operator is applied and equation (1) is substituted into it, the result is 


$$
y(t+1)=C A x(t)+\sum_{i=1}^{m} C B_{i} x(t) u_{i}(t)
$$

Equations (1) and (5) taken together give a pair of algebraic equations relating $x(t+1), x(t), y(t+1)$ and $u(t)=\left[u_{1}(t) \ldots u_{m}(t)\right]^{\mathrm{T}}$

$$
\left[\begin{array}{cccc}
I_{n} & -B_{1} x(t) & \ldots & -B_{m} x(t) \\
0 & C B_{1} x(t) & \ldots & C B_{m} x(t)
\end{array}\right]\left[\begin{array}{c}
x(t+1) \\
u(t)
\end{array}\right]=\left[\begin{array}{c}
A x(t) \\
y(t+1)-C A x(t)
\end{array}\right] .
$$

The desired inverse system is a pair of equations for $x(t+1)$ and $u(t)$ in terms of $x(t)$ and $y(t+1)$. It is known that the equations (6) can be solved for $x(t+1), u(t)$ uniquely if and only if the matrix

$$
R=\left[\begin{array}{cccc}
I_{n} & -B_{1} x(t) & \ldots & -B_{m} x(t) \\
0 & C B_{1} x(t) & \ldots & C B_{m} x(t)
\end{array}\right]
$$

has an inverse. Now consider the following fact in matrix theory.

Le m ma $\left.1{ }^{[4}\right]$. Any $n$-dimensional square matrix of rank one can be uniquely (within a scalar factor) expressed as a product of a column and a row $n$-vector.

Consequently, the matrices $B_{i}$ can be expressed as

$$
B_{i}=g_{i} h_{i}^{\mathrm{T}}, \quad i=1, \ldots, m .
$$

Let us introduce notations

$$
h_{i}^{\top} x(t)=\gamma_{i}(x), \quad \Gamma(x)=\operatorname{diag}\left\{\gamma_{1}(x) \ldots \gamma_{m}(x)\right\},
$$

In these notations

$$
G=\left[g_{1} \ldots g_{m}\right]
$$

$$
R=\left[\begin{array}{cc}
I_{n} & -G \Gamma(x) \\
0 & C G \Gamma(x)
\end{array}\right]
$$

The matrix $R$ has an inverse if and only if the matrix $C G$ has a full rank and

$$
\gamma_{i}(x)=h_{i}^{\mathrm{T}} x(t) \neq 0, \quad i=1, \ldots, m, \quad t=0,1, \ldots .
$$

If the matrix $R$ is invertible, then applying the well-known matrix inversion lemma, we get

$$
R^{-1}=\left[\begin{array}{cc}
I_{n} & G(C G)^{-1} \\
0 & \Gamma^{-1}(x)(C G)^{-1}
\end{array}\right]
$$

and the solution of equations (6) is

$$
\begin{aligned}
& x(t+1)=\left[I-G(C G)^{-1} C\right] A x(t)+G\left(C^{\prime} G\right)^{-1} y(t+1), \\
& u(t)=-\Gamma^{-1}(x)(C G)^{-1} C A x(t)+\Gamma_{m}^{-1}(x)(C G)^{-1} y(t+1) .
\end{aligned}
$$

Now, denote $H_{i}=\left\{x \mid h_{i}^{T} x=0\right\}, X=R^{n}-\bigcup_{i=1}^{m} H_{i}$. For any $x\left(t_{0}\right) \in X$ introduce a set $u_{\substack{t_{0}, t_{1} \\ x\left(t_{0}\right)}}^{t_{0}}$ containing the sequences $\left\{u\left(t_{0}\right), u\left(t_{0}+1\right), \ldots, u\left(t_{1}\right)\right\}$ that yield $x(t) \in X, \quad t=t_{0}, \ldots, t_{1}$.

We have proved the following theorem.

Theorem 1. The MIMO BLS (1)-(3) has the inverse on the time interval $\left[t_{0}, t_{1}\right]$ at $x\left(t_{0}\right) \in X$ with respect to $\left\{u\left(t_{0}\right), \ldots, u\left(t_{1}\right)\right\} \in \mathcal{U}_{x\left(t_{0}\right)}^{t_{0}, t_{1}}$ if and only if the matrix CG has a full rank. If the BLS (1)-(3) has the inverse, then the inverse system is a linear system with nonlinear output defined by 


$$
\begin{aligned}
x(t+1) & =\widetilde{A} x(t)+\widetilde{B} y(t+1), \\
u(t) & =\widetilde{C} x(t)+\widetilde{D} y(t+1),
\end{aligned}
$$

where

$$
\begin{aligned}
& \widetilde{A}=\left[I-G(C G)^{-1} C\right] A, \\
& \widetilde{B}=G(C G)^{-1}, \\
& \widetilde{C}=-\Gamma^{-1}(x)(C G)^{-1} C A, \\
& \widetilde{D}=\Gamma^{-1}(x)(C G)^{-1} .
\end{aligned}
$$

4. Controllability of inverse system. A theorem will be presented that gives sufficient conditions for the complete controllability of the inverse system.

Theorem 2. If the MIMO BLS (1)-(3) is completely controllable, then the inverse system (7) is completely controllable.

Proof. The proof of this theorem is based on Lemma 2 given by P. Hollis and D. N.P. Murthy $\left[{ }^{5}\right]$.

Le mma 2. If the BLS (1)-(3) is completely controllable, then rank

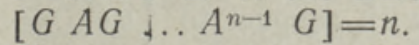

The inverse system of (1)-(3), which is a time-invariant linear system by Theorem 1 , is completely controllable if and only if rank $[\widetilde{B} \widetilde{A} \widetilde{B} \cdot$. . $\left.\ldots \widetilde{A}^{n-1} \widetilde{B}\right]=n$. Using the relationship between $A, \widetilde{B}$ and $A, G, C$ given by Theorem 1, the matrix $\left[\widetilde{B} \widetilde{A} \widetilde{B} \ldots \widetilde{A}^{n-1} \widetilde{B}\right]$ can be expressed as

$$
\left[\begin{array}{lllll}
\widetilde{B} & \widetilde{A} & \ldots & \widetilde{A}^{n-1} & \widetilde{B}
\end{array}\right]=\left[\begin{array}{llll}
G & A G \ldots A^{n-1} & G
\end{array}\right] T,
$$

where $T$ is $n m \times n m$ upper triangular block Toeplitz matrix with the matrices $(C G)^{-1}$ on the main diagonal. It follows, therefore, that the matrix $T$ has full rank and

$$
\operatorname{rank}\left[\begin{array}{lllll}
\widetilde{B} & \widetilde{A} & \ldots & \widetilde{A}^{n-1} & \widetilde{B}
\end{array}\right]=\operatorname{rank}\left[\begin{array}{llll}
G & A G \quad \therefore A^{n-1} & G
\end{array}\right]
$$

Applying. Lemma 2, Theorem 2 follows.

5. Example. Consider the bilinear system

$$
\begin{aligned}
x(t+1) & =A x(t)+B_{1} x(t) u_{1}(t)+B_{2} x(t) u_{2}(t) \\
y(t) & =C x(t),
\end{aligned}
$$

where

$$
\begin{array}{cc}
A=\left[\begin{array}{lll}
1 & 0 & 0 \\
0 & 0 & 1 \\
0 & 0 & 0
\end{array}\right], \quad B_{1}=\left[\begin{array}{lll}
0 & 0 & 0 \\
0 & 1 & 0 \\
0 & 0 & 0
\end{array}\right], \quad B_{2}=\left[\begin{array}{rrr}
0 & -1 & 0 \\
0 & 0 & 0 \\
0 & 1 & 0
\end{array}\right], \\
C=\left[\begin{array}{lll}
1 & 0 & 0 \\
0 & 1 & 0
\end{array}\right]
\end{array}
$$

We have

$$
B_{1}=g_{1} h_{1}^{\mathrm{T}}=\left[\begin{array}{l}
0 \\
1 \\
0
\end{array}\right]^{\left[\begin{array}{lll}
0 & 1 & 0
\end{array}\right]}, \quad B_{2}=g_{2} h_{2}^{\mathrm{T}}=\left[\begin{array}{r}
1 \\
0 \\
-1
\end{array}\right]^{\left[\begin{array}{lll}
0 & -1 & 0
\end{array}\right]} .
$$

The matrix $C G=\left[\begin{array}{ll}0 & 1 \\ 1 & 0\end{array}\right]$ has full rank. To construct the inverse system for (8) using Theorem 1, we evaluate 


$$
\begin{aligned}
& \tilde{A}=\left[I-G(C G)^{-1} C\right] A=\left[\begin{array}{lll}
0 & 0 & 0 \\
0 & 0 & 0 \\
1 & 0 & 0
\end{array}\right], \\
& \widetilde{B}=G(C G)^{-1}=\left[\begin{array}{rr}
1 & 0 \\
0 & 1 \\
-1 & 0
\end{array}\right], \\
& \widetilde{C}=-\Gamma^{-1}(x)(C G)^{-1} C A=\left[\begin{array}{ccc}
0 & 0 & -1 / x_{2} \\
1 / x_{2} & 0 & 0
\end{array}\right], \\
& \widetilde{D}=\Gamma^{-1}(x)(C G)^{-1}=\left[\begin{array}{cc}
0 & 1 / x_{2} \\
-1 / x_{2} & 0
\end{array}\right] .
\end{aligned}
$$

The system $(8)$ is invertible at $x(0)=\left[\begin{array}{lll}0 & 1 & 0\end{array}\right]^{\mathrm{T}}$ with respect to $\{u(0)=$ $\left.=\left(\begin{array}{l}1 \\ 2\end{array}\right), u(1)=\left(\begin{array}{l}0 \\ 1\end{array}\right), u(2)=\left(\begin{array}{l}1 \\ 0\end{array}\right), u(3)=\left(\begin{array}{r}-1 \\ 0\end{array}\right)\right\}$ on the time interval $[0,3]$, but it is not invertible at the same $x(0)$ with respect to $\{u(0)=$ $\left.=\left(\begin{array}{l}1 \\ 2\end{array}\right), \quad u(1)=\left(\begin{array}{l}0 \\ 1\end{array}\right), \quad u(2)=\left(\begin{array}{l}1 \\ 0\end{array}\right), \quad u(3)=\left(\begin{array}{l}0 \\ 1\end{array}\right)\right\}, \quad$ because then $h_{1}^{\mathrm{T}} x(4)=h_{2}^{\mathrm{T}} x(4)=0$.

\section{REFERENCES}

1. Hirschorn, R. M. SIAM J. Contr. and Opt., 15, № 6, 1039-1049 (1977),

2. Hirschorn, R. M. SIAM J. Contr. and Opt., 17, № 2, 289-297 (1979).

3. Hirschorn, R. M. IEEE Trans. Automat. Contr., 24, № 6, 855-865 (1979).

4. Hsu, C. S., Mohler, R. R. J. Dyn. Syst., Measurem. and Contr., 102, № 2, 103-105 (1981).

5. Hollis, P., Murthy, D. N. P. Int. J. Syst. Sci., 12, № 4, 485-494 (1981).

Academy of Sciences of the Estonian SSR, Institute of Cybernetics 